\title{
Evaluation and calculations of modes resonant cantilever for laser optical fiber assembly
}

\author{
Mohanad Aljanabi \\ Electrical Power Techniques Engineering, Technical College /AL- Mausaib, Al-Furat Al-Awsat Technical University \\ Najaf, Iraq.
}

\begin{tabular}{l} 
Article Info \\
\hline Article history: \\
Received Jan 10, 2019 \\
Revised May 1, 2019 \\
Accepted Jul 1, 2019 \\
\hline
\end{tabular}

Keywords:

Ansys

Cantilever

Geometry of the Assembly

Laser

Modes Resonant Frequency

\begin{abstract}
Biosensors depend on cantilevers have developed a promising tool for detecting biomedical, optical laser communication and many fields of interactions with high accuracy. We modeled the operation of cantilevers with two magnetic and coil using Ansys program. This simulation technique can capably be used to select the appropriate design and dimensions of cantilever with the geometry of system. The primary main of the magnetic design is to improve the geometry of the coil and shape to yield a highly uniform for 3D of optical fiber includes Silica Glass and Nickel cantilever, two magnets and coil that apply to force on the cantilever cylinder is using as a cantilever in the designing of this case. Results from many assessments which usage micro-cantilever have made known that these biosensors can demonstrate good compassion and high resolve. Additionally, for smart materials will regulator the strategy in the associations of reducing the vibrations amplitude and frequency to development the effectiveness of the designer in the new uses. Purposes to this problem comprise modification the approaches of numbers the resonant frequency to yield by cantilivier after the signal pass from laser system. The experimental of mode resonant frequency $\left(f_{n}\right)$ of $3933.1594 \mathrm{~Hz}$ illustrations reasonable agreement with the Comsol model being within $10 \%$ of the expected result.
\end{abstract}

Copyright (C) 2020 Institute of Advanced Engineering and Science. All rights reserved.

\section{Corresponding Author:}

Mohanad Aljanabi,

Electrical Power Techniques Engineering,

Technical College /AL- Mausaib, Al-Furat Al-Awsat Technical University, Najaf, Iraq.

Tel: 009647735401438

Email: mhn@atu.edu.iq

\section{INTRODUCTION}

A cantilever is a beam supported on only one end. The shaft transfers the load to the support where it has managed the moment of force and shear stress. The magnetic field distribution $(\mathrm{H})$ from two permanent magnets was demonstrated to support in the design of a magnet system for optical fibre laser studies to be achieved in the Laser-Matter Interactions Laboratory [1]. Cantilevers were presented as a sensing element of different recognition systems because of their high sensitivity. The chemical detection in the air has been confirmed in resonant frequencies [2,3]. Currently developing small and inexpensive methods for monitoring various analysis and calculations of the resonance frequency in laser optical detection for cantilever with two magnetic and coil factors is one of the increasing demands in life science [2].

Cantilever based sensors are being studied for their high sensitivity and in assortments of requests by many researchers [4]. They are a sensitive deflection or modification in the numbers of mode frequency sensors. Cantilever sensors are presently utilized in two basically different methods, which may be represented as a static mode and dynamic (resonant) mode. The static method of procedure holds same promising potential for biomedical requests [5]. 
The bending and resonant frequency shift of the cantilever can be measured with high accuracy using optical fibre reflection method. One of the advantages of the cantilever method is that both bending and resonant frequency can be measured in a single measurement [6]. Dynamic cantilevers are normally not appropriate for application in which the sensors should be placed in an aqueous environment. This is because the damping outcome in resolution can move cantilever's resonance frequency as shown in Figure 1 . In this situation, to have a sensing system with high solution, it might be important to have an identical complicated the format designer [7] as shown in Figure1. As the spectral locations of the resonance frequencies are sure of the cantilever geometry, material types and mass, a modification in mass will origin a shift in resonance frequency. By way of the sizes of the cantilever's reduction, the sensitivity rises, suggesting that resonant cantilever sensors have the future potential of distinguishing the mass of different molecules [8]. Some approaches have been advanced to actuate cantilevers and detect the resonant motion. The utmost usually used are designated in the review article by Stemme [9] and comprise electrostatic actuation and optical fibre laser detection [8].

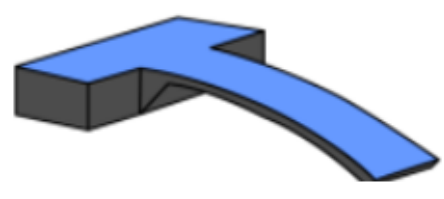

(a)

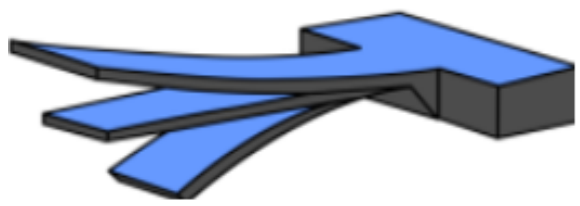

(b)

Figure1. Resonant frequencies of cantilever (a) Static, (b) dynamic [8].

Optical sensors typically study depend on emitting light to the surface of the cantilever with a laser and analysing the location of reflected light to sensitive the beam movement [10]. Figure 2 shows the optical detection for cantilever technique.

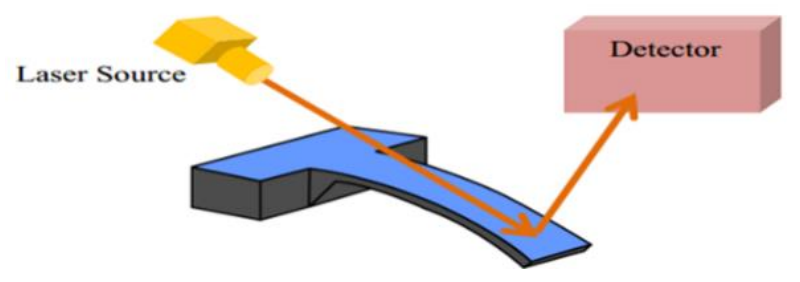

Figure 2. Laser source for cantilevers detection system [10].

However, several studies projected utilizing multiplexing technique to decrease the numeral of sources and detectors. In 2012 a set advanced a system for monitoring eight cantilevers [11]. This format involves of 8 Vertical-cavity surface-emitting laser and photodetector. Correspondingly, it is probable to utilize one CCD image screen as a detector for wholly biosensors. Yue et al. utilise one collimated beam as a light source for 100 of cantilevers and distinguish their difference with a CCD plane [11]. In addition, taking high sensitivity and respectable determination, another benefit of optical laser detection is the ease of the fabrication procedure for sensors which are going to be utilised with an optical laser arrangement. For any cantilever sensor, there are two properties of the frequency response that are of central importance: the position of the resonance frequency $\left(f_{n}\right)$ and Q-factor, given by $Q=\Delta f / f 0$, where $\Delta f$ is the FWHM of the resonance peak $[12,13]$. But, subsequently detection in these instruments will be completed depend on light's reflection from a surface, it is significant to have a uniform surface to evade light scattering [14]. Now that the magnetic flux density around the magnets is characterized, dynamic magnetic analysis can be performed to determine how much voltage is expected to be generated with the chosen winding configuration. The voltage generated by a coil is the derivative of the flux linkage with respect to time [15].

To narrow down the scope of the analysis, the general configuration of the magnets and the coils are determined first. The simplest way to increase the change in magnetic flux is to use a strong magnet. Another way to achieve the same is to use two different magnets that are attached side-by-side with different dimensions $[16,17]$. 


\section{METHOD}

In an electromagnetic field, two permanent magnets are utilizing to yield a strong magnetic field and coil is utilizing as the conductor with the vibration of the cantilever. In Figure 1, the magnetic field $(\mathrm{H})$ is uniform. The $\mathrm{H}$ cut by the coil varies with the relative displacement between 2 magnets and the coil. The emf is assumed by [18]:

$$
\mathrm{emf}=-\mathrm{NlB} \frac{\mathrm{dz}}{\mathrm{dt}}
$$

$\mathrm{B}$ is the flux density going through the coil, and dz/dt is the relative velocity between the 2magnents and the coil with the vibration of the cantilever with passes the light from a laser. For Figure 1 , the $\mathrm{H}$ varies with the distance apart from the 2-magnets and the emf is given by [19, 20]:

$$
\mathrm{emf}=-\mathrm{NB} \frac{\mathrm{dB}}{\mathrm{dt}} \frac{\mathrm{dz}}{\mathrm{dt}}
$$

Where $S$ is the active area of the coil and $\mathrm{dB} / \mathrm{dz}$. For an unassuming elastic beam problem with uniform cross-sectional area, a recognized normal frequency can be designed by:

$$
\mathrm{Wn}=1 / 2 \pi(\beta \mathrm{l}) 2(\mathrm{EI} / \rho \mathrm{Al} 4)
$$

Where A and I are the area of cross-section and the length of the flexible beam, $\beta 1$ is a constant relative to the vibration bound state. The constant $\beta 1$ for five resonant frequencies of a cantilever shape 14.41526e5, $4.279887 \mathrm{e} 5,1.93684 \mathrm{e} 5,1.09385 \mathrm{e} 5$ and $68754.4271 \mathrm{~Hz}$ respectively. EI is the equivalent bending stiffness. Cantilevers are attractive as sensors because they make available easy and good sensing with high sensitivity $[21,22]$.

\section{RESULTS AND SIMULATIONS}

The goal of our simulations, evaluations and calculations is to design the system for modes of resonant frequencies for Laser optical fiber biosensors assembly with vibration of the cantilever beam by apply the laser source for this system, which can measure the numbers modes of vibration when the signal pass from laser source. The biosensor is implemented by using optical fiber transmitter, receiver group, oscilloscope, cantilever, power supply, (laser or IR) source, two permanent magnets have the same size and shape and one-cylinder coil and AC source. The principle of this study that when the magnetic field distribution of the permanent magnet to produce a force, this force performances on the cantilever beam accordingly, the cantilever will vibrate with modes of a resonance frequency as shown in Figure 3.

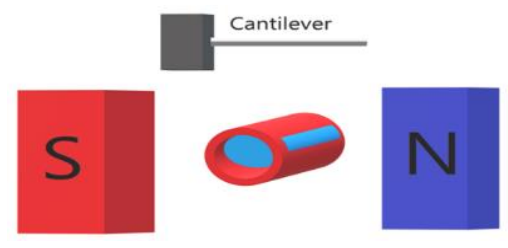

Figure 3. Schematic view of the 2- permanent magnetic and one-cylinder coil with coating Ni; vibration of cantilever beam [23].

The magnetic actuation of were located in parallel to each additional in such a technique to form a DC magnetic field lines $(\mathrm{H})$ in the direction of the beam. $\mathrm{H}$ varieties magnetic moments in the coating cantilever along the DC field. After the cantilever is animated by the AC magnetic field designed by the coil effective, the $\mathrm{H}$ attempt to make even themselves with the AC field exciting the beam at the frequency of the AC magnetic flux.

Silica Glass cylinder is using as a cantilever in the designing of this case, where outer radius $\mathrm{r} 1=62.5 \mathrm{e}-3 \mathrm{~mm}$, inner radius $\mathrm{r} 2=25 \mathrm{e} 3 \mathrm{~mm}$, length $\mathrm{L}=5 \mathrm{~mm}, \mathrm{E}=73.1 \mathrm{e} 9 \mathrm{~Pa}, \rho=2203(\mathrm{~kg} \backslash \mathrm{m} 3)$ and $\sigma=0.17$, analytically we can find the frequency of vibration mode from the equations below:

$$
f=\frac{3.52}{2 \pi l^{2}} \sqrt{\frac{E I}{\rho A}}
$$




$$
I=\frac{\pi d^{4}}{64}
$$

Where I refer to of the second moment of area. By substitution (5) in (4) we get:

$$
\begin{aligned}
& f=\frac{3.52 d}{8 \pi l^{2}} \sqrt{\frac{E}{\rho}} \\
& E=\frac{64 \pi^{2} \rho l^{4} f_{n}^{2}}{m_{n}^{4} d^{2}} \\
& f_{n}=\frac{m_{n}^{2} d}{8 \pi l^{2}} \sqrt{E / \rho}
\end{aligned}
$$

The cantilever utilized in this study is fabricated utilizing an easy, single mask process utilising many stages [24-26]. Using Ansys code of the research presents the design, simulation as shown in Figure 4(a\&b) shows the coil between two magnets in 3D. The numbers of mode vibration with and without coating the cantilivier as see in Table 1. Moreover, the force acting can be calculated analytically by the equations of the magnetic field as follows:

$$
\begin{aligned}
& F_{B}=I l \times B \\
& B=\mu_{o} H=\mu_{o}\left(\frac{N}{L}\right) I
\end{aligned}
$$

By substitution we get:

$$
F_{B}=I^{2} l \times \frac{\mu_{o}}{L}
$$

Where; $F_{B}$ : Force $(\mathrm{N}), I$ : current (Amp), l: length of wire $(\mathrm{m}), L$ : length of coil $(\mathrm{m}), \mathrm{N}$ : number of turns. $B$ : Magnetic flux density (weblm $\left.{ }^{2}\right),(\mathrm{T}), \mu_{o}$ : permeability of free space $\left(\mathrm{N} \backslash \mathrm{A}^{2}\right)$, $\mathrm{H}$ : magnetic field (Alm). The PLANE13 was utilised in the permanent magnets, the permeable air and the iron material regions. We have similarly designed the $f_{n}$ by using (6) and Table 2 gets the results from simulation and theoretical designs. As can be seen in this table, both values are in good agreement with each other [27].

The experimental $f_{n}$ of $3933.1594 \mathrm{~Hz}$ illustrations reasonable agreement with the Comsol model being within $10 \%$ of the expected result. The values of resonance frequency from the experiment are being compared with the simulation results [28]. Simulation and experimental values are very close to each other. For analytical calculations we used mode of vibration with and without coating the cantilivier as see in Table 1. From equations leading electromagnetic field the generated energy reductions with device parameters and reducing input harmonics vibration [28-30]. Then Figure 5 appears simulation of two magnets and coil in 2D. Figure 6 shows that simulation of two magnets and coil in 2D when the applied magnetic field for the box in the air.

In Ansys program, the force between two magnets had been simulated in 3D as shown in Figure 7 file code Ansys force between two per magnet. Figure 8 shows the distribution of the magnetic flux density in the final design of the system.

Table 1. Modes of vibration with and without coating the cantilivier

\begin{tabular}{cccc} 
Values of frequency without coating & $25153.2307 \mathrm{~Hz}$ & $1.557336 \mathrm{e} 5 \mathrm{~Hz}$ & $4.279887 \mathrm{e} 5 \mathrm{~Hz}$ \\
\hline Values of frequency with coating by Ni & $25153.479 \mathrm{~Hz}$ & $1.557351 \mathrm{e} 5 \mathrm{~Hz}$ & $4.279886 \mathrm{e} 5 \mathrm{~Hz}$ \\
\hline
\end{tabular}

Table 2. The results and calculations of modes in resonance frequencies of the system

\begin{tabular}{lllllll}
\hline $\mathrm{L}(\mathrm{mm})$ & $\mathrm{F}_{1}(\mathrm{~Hz})$ Equation & $\mathrm{F}_{1}(\mathrm{~Hz})$ Ansys & $\mathrm{F} 2(\mathrm{~Hz})$ Equation & $\mathrm{F}_{2}(\mathrm{~Hz})$ Ansys & $\mathrm{F}_{3}(\mathrm{~Hz})$ Equation & $\mathrm{F}_{3}(\mathrm{~Hz})$ Ansys \\
\hline 1 & $1.008994 \mathrm{e} 5$ & $1.000763 \mathrm{e} 5$ & $6.41315 \mathrm{e} 5$ & $5.989104 \mathrm{e} 5$ & $17.678104 \mathrm{e} 5$ & $14.41526 \mathrm{e} 5$ \\
2 & 25224.617 & 25153.2307 & $1.603288 \mathrm{e} 5$ & $1.557336 \mathrm{e} 5$ & $4.41952602 \mathrm{e} 5$ & $4.279887 \mathrm{e} 5$ \\
3 & 11210.938 & 11192.0518 & 71257.26 & 69762.978 & $1.9642337 \mathrm{e} 5$ & $1.93684 \mathrm{e} 5$ \\
4 & 6306.152 & 6255.3218 & 40082.208 & 39177.8604 & $1.1048815 \mathrm{e} 5$ & $1.09385 \mathrm{e} 5$ \\
5 & 4035.9378 & 3933.1594 & 25652.613 & 24688.061 & 70712.416 & 68754.4271 \\
\hline
\end{tabular}




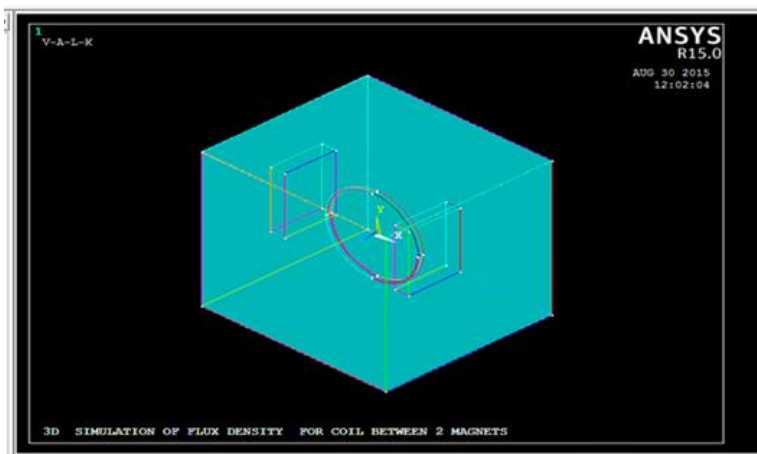

Figure 4(a). Simulation of the flux density in 3D

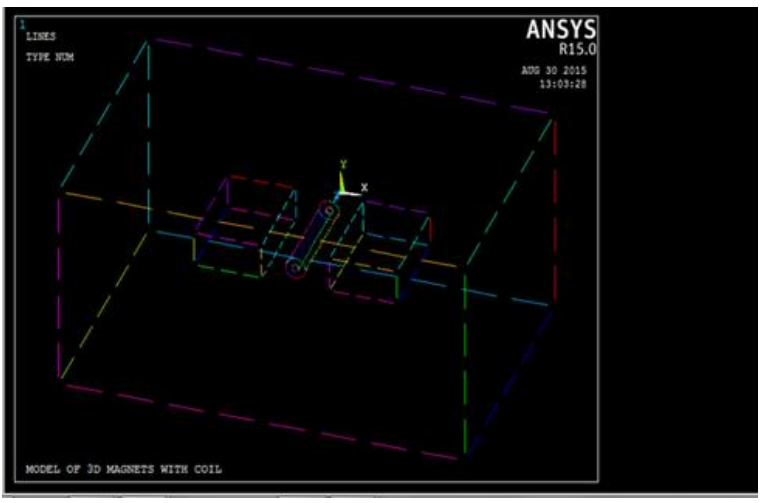

Figure 5. Simulation of two magnets and cylinder coil in 2D

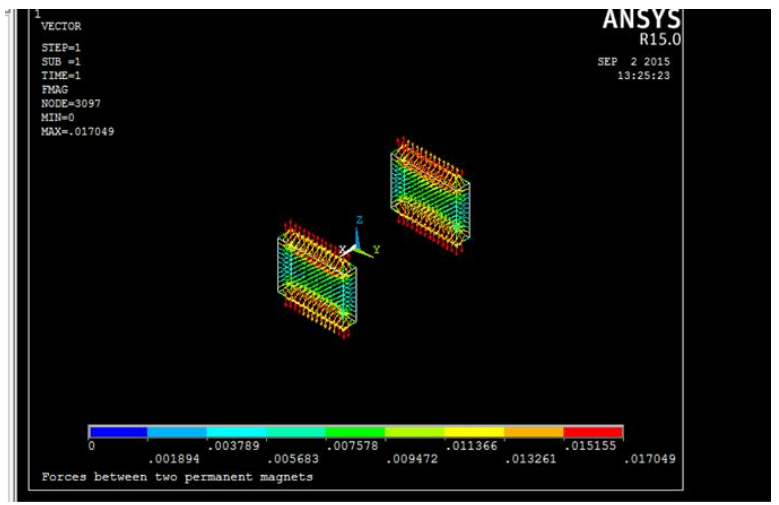

Figure 7. Magnetic force distribution in the magnets

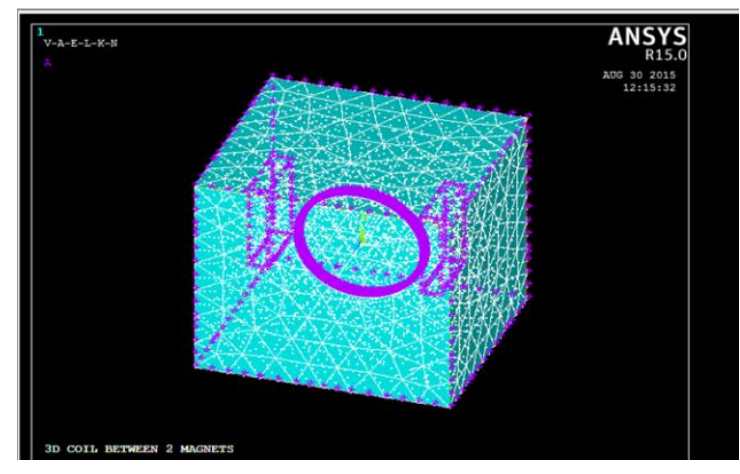

Figure 4(b). 3D simulation of two-pole configuration

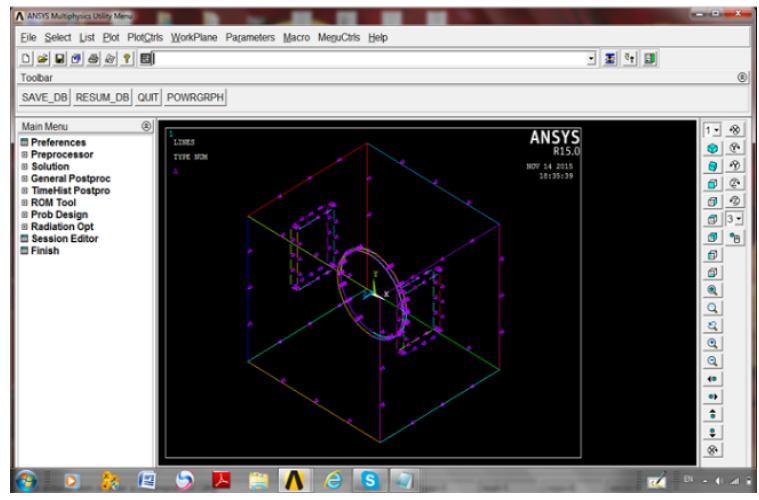

Figure 6. Simulation of two magnets and coil in 2D when the applied magnetic field for the box in the air

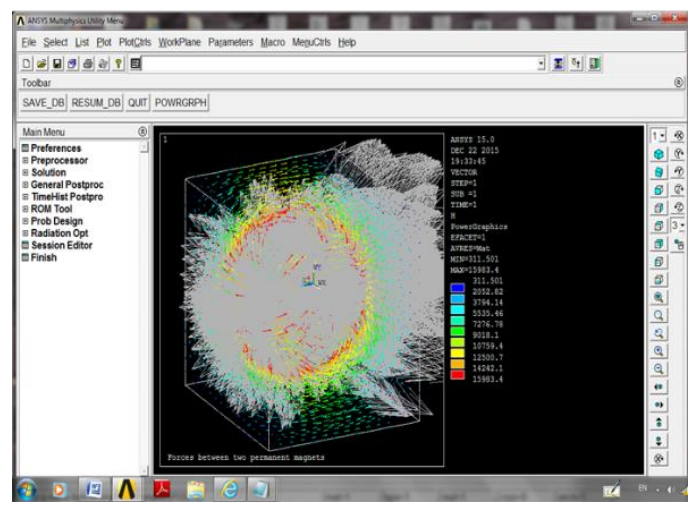

Figure 8. Distribution of $\mathrm{H}$ in the final design of the system

\section{CONCLUSION}

The methods of modification of the resonant frequency contain varying the sizes of the cantilever and two magnets with variable coil conditional on the altering dimensions, supplies of the cantilever, source apply (laser) with assembly system [31]. Results from many assessments which usage micro-cantilever have made known that these biosensors can demonstrate good compassion and high resolve. Additionally, for smart materials will regulator the strategy in the associations of reducing the vibrations amplitude and frequency to development the effectiveness of the designer in the new uses. This system is appropriate for low-price with movable laser biosensors assembly to become the right new locations of detection. 
Modern studies, we will improvement the detection truth by an increase the numbers of turns coil and progress the information by rising the standards of numbers in modes of frequencies. The biosensor technology and smart materials complete are significant as it is mountable for instantaneous for several biomedical devices and laser optical fiber, communication for approximately sensitive biosensors system.

When compare this method show less time uncontrollable and cost real, in comparison to various extra alike applications. This system is identical simple to implement, a varied range of frequencies, dependability and accuracy to get good results.

Nevertheless, the modes of resonance frequency can influence of $68754.4271 \mathrm{~Hz}$ for five mode and the shift $\mathrm{o} / \mathrm{p}$ is enlarged by approximately $34.21 \%$ despite the fact 0.54 times of the critical system is useful of force applied [32].

\section{REFERENCES}

[1] http://aries.ucsd.edu/LASERLAB.

[2] E.A. Wachter, T. Thundat, "Micromechanical sensors for chemical and physical measurements", Rev. Sci. Instrum. 66 (1995) 6, http://dx.doi.org/10.1063/1. 1145484.

[3] T.P.I. Thundat, R.J.W. Oden, "Microcantilever sensors, Microscale Thermophys". Eng. 1 (1997) 185-199, http://dx.doi.org/10.1080/108939597200214.

[4] M. Yue, A. Majumdar, and T. Thunder, "Cantilever arrays: A universal platform for multiplexed label-free bioassays," in BioMEMS and Biomedical Nanotechnology (M. Ferrari, R. Bashir, and S. Wereley, eds.), pp. 21-33, Springer US, (2007). 10.1007/978-0-387-25845-42.

[5] Michael Sepaniak, Panos daeskos, Nickolay Lavrik, Christopher Tipple, "Microcantilever Transducers: A New Approach in Sensor technology", Analytical Chemistry, November 1, (2002). pp. 568A-575A.

[6] Allen, M. G. (1994)." Design, fabrication, and application of magnetic micro actuators. Micro Machine and Human Science", 5. C, Z. (2004).

[7] Mahalik, N.P. Micromanufacturing and nanotechnology. Springer, (2006)

[8] C, Z.," Cantilever-based biosensors. Analytical and Bio Analytical Chemistry", (2014), pp.946-959.

[9] Z.J. Davis, G. Abadal, B. Helbo, O. Hansen, F. Campabadal, F. Perez Murano, J. Esteve, E. Figueres, J. Verd, N. Barniol, and A. Boisen." Monolithic integration of mass sensing nano-cantilevers with CMOS circuitry. Sensors and Actuators A: Physical", 105(3):311-319, (2013).

[10] Göran Stemme. "Resonant silicon sensors. Journal of Micromechanics and Microengineering", 1(2):113-125, (1991).

[11] S. L. Biswal, "Micro and- nanocantilever systems for molecular analysis," in Nano and Microsensors for Chemical and Biological Terrorism Surveillance, J. B.-H. Tok, Ed., RSC Publishing, (2008), pp. 82-97.

[12] M. Yue et al., "A 2-D microcantilever array for multiplexed biomolecular analysis," Journal of Microelectromechanical Systems, vol. 13, pp. 290-299, (2004).

[13] Z.J. Davis, G. Abadal, B. Helbo, O. Hansen, F. Campabadal, F. Perez Murano, J. Esteve, E. Figueras, J. Verd, N. Barniol, and A. Boisen. "Monolithic integration of mass sensing nano-cantilevers with CMOS circuitry. Sensors and Actuators A: Physical", 105(3):311-319, (2013).

[14] F. Shen, P. Lu, S.J. O'Shea, K.H. Lee, and T.Y. Ng. "Thermal effects on coated resonant microcantilevers. Sensors and Actuators A: Physical, 95(1):17-23, (2017).

[15] P. Wang et al. "A micro electromagnetic low-level vibration energy harvester based on MEMS technology," Microsyst. Technol., vol. 15, no. 6, pp. 941-951. (2014).

[16] C. T. Pan et. al. "Fabrication and analysis of a magnetic self-power microgenerator," J. Mag. Magn. Mater., vol. 304, no. 1, pp. e394-e396, (2006).

[17] Uzairue Stanley, Victor Matthews Olu, Charles Ochonogor, Amaize Peter, Anyasi Francis, "Experimental Analysis of Cable Distance Effect on Signal Attenuation in Single and Multimode Fiber Optics", International Journal of Electrical and Computer Engineering (IJECE) Vol.8, No.3, June (2018), pp.1577 1582, ISSN: 2088-8708, DOI: 10.11591/ijece. v8i3. pp. 1577-1582.

[18] A. Boisen et al., "Cantilever-like micromechanical sensors," Reports on Progress in Physics, vol. 74, pp. 036101, (2011).

[19] K. M. Hansen and T. Thundat, "Microcantilever biosensors," Methods, vol. 37, pp. 57-64, (2005).

[20] D. de Bhail'1s, C. M., "Modelling and analysis of a magnetic micro actuator, Sensors and Actuators", pp. 285-289, (2010).

[21] Yuvaraja M., Senthil Kumar M., "Active Vibration Control of SMART Composite structure - A Review”, National Journal of Technology 6: 27 - 24, (2010).

[22] J. W. Chamberlain and D. M. Ratner, "Label-Free Biosensors for Biomedical Applications: The Potential of Integrated Optical Biosensors and Silicon Photonics," in Optical, Acoustic, Magnetic, and Mechanical Sensor Technologies, K. Iniewski, Ed., CRC Press, (2012), pp. 45-78.

[23] Mohanad Aljanabi," Resonance Frequency Analysis of Laser Optical Fiber based on Microcantilever", International Indonesian Journal of Electrical Engineering and Computer Science Vol 9, No 4: August 2019(part II). 
[24] O. Cakmak, N. Kilinc, E. Ermek, A. Mostafazadeh, C. Elbuken, G.G. Yaralioglu, et al., "LoC sensor array platform for real-time coagulation measurements, Micro Electro Mech. Syst. (MEMS)", (2014) IEEE 27th Int. Conf. (2014) 330-333, http://dx.doi.org/10.1109/MEMSYS.2014.6765643.

[25] O. Cakmak, C. Elbuken, E. Ermek, A. Mostafazadeh, I. Baris, B. Erdem Alaca, et al., "Microcantilever based disposable viscosity sensor for serum and blood plasma measurements, Methods 63" (2013) 225-232, http://dx.doi.org/10. 1016/j.ymeth.2013.07.009.

[26] O. Cakmak, E. Ermek, N. Kilinc, S. Bulut, I. Baris, I.H. Kavakli, et al.," A cartridge-based sensor array platform for multiple coagulation measurements from plasma", Lab Chip 15 (2015) 113-120, http://dx.doi.org/10.1039/C4LC00809J.

[27] I Bouneb, F Kerrour, "Nanometric Modelization of Gas Structure, Multidimensional using COMSOL Software", International Journal of Electrical and Computer Engineering (IJECE) Vol.8, No.4, August (2018), pp.2014 2020, ISSN: 2088-8708, DOI: 10.11591/ijece. v8i4. pp. 2014-2020.

[28] Mechanical simulation with Ansys workbench, Ansys Inc.; http:// www: Ansys .com/ solutions / simulationenvironment .asp.

[29] Ching N H, Wong H Y, Li W J, Leong P H W and Wen Z, " A laser micromachined vibrational to electrical power transducer for wireless sensing systems Sensors Actuators", A 97-98 685-90, (2015).

[30] Md Rabiul Awal, Muzammil Jusoh, R. Badlishah Ahmad, Thennarasan Sabapathy, M. Najib M. Yasin, Mohd Hafizuddin Mat," Designing cantilever dimension for low power wireless applications", Indonesian Journal of Electrical Engineering and Computer Science Vol. 14, No. 2, May (2019), pp. 758 764 ISSN: 2502-4752, DOI: 10.11591/ijeecs. v14.i2. pp758-764.

[31] HuiNee Ow, Muataz H. Salih, ChinBeng Lim," Design and implementation of laser based intelligent embedded dual modes cruise control system using FPGA", Indonesian Journal of Electrical Engineering and Computer Science Vol 13, No 3: March (2019).

[32] Heng Chen, Jun-Shan Wang , Chao Chen , Shi-xiang Liu , and Hai-Peng Chen," Dynamic Modeling and Characteristic Analysis of Cantilever Piezoelectric Bimorph", Hindawi, Mathematical Problems in Engineering, Volume (2019), Article ID 3926906, 9 pages, https://doi.org/10.1155/2019/3926906 